\title{
Erratum to: $\mathrm{O}$-antigen polymerase adopts a distributive mechanism for lipopolysaccharide biosynthesis
}

\author{
Guohui Zhao • Baolin Wu $\cdot$ Lei Li $•$ Peng George Wang
}

Published online: 2 April 2014

(C) Springer-Verlag Berlin Heidelberg 2014

Erratum to: Appl Microbiol Biotechnol (2014)

DOI 10.1007/s00253-014-5552-7

The original version of this article inadvertently contained mistakes. In the discussion part, when we discussed the "catch-and-release" mechanism, the cited paper (Islam et al., eBio, 2010) is not correct. It should be another publication (Islam et al., JBC 2011) from the same lab.

The second error is that we mentioned that PL3 and PL5 of WzyPa are proposed to carry net-negative and net-positive charges, respectively. In fact, the opposite is correct. In Islam et al. (2011), they characterized PL3 (pI 8.59) and PL5 (pI 5.49) of WzyPa and proposed that PL3 should be netpositively charged (to recruit a net-negatively-charged lipidlinked single $\mathrm{O}$ unit in this organism) under physiological conditions.

The online version of the original article can be found at http://dx.doi.org/ 10.1007/s00253-014-5552-7.

G. Zhao $\cdot$ B. Wu $\cdot$ L. Li $\cdot$ P. G. Wang $(\bowtie)$

Center for Diagnostics and Therapeutics and Department of Chemistry, Georgia State University, Atlanta, GA 30303, USA

e-mail: pwang11@gsu.edu 